\title{
Naturaleza de la ciencia en noticias científicas de la prensa: análisis del contenido y potencialidades didácticas
}

\section{The nature of science in press articles about science: con- tent analysis and pædagogical potential}

\author{
Antonio García-Carmona \\ Departamento de Didáctica de las Ciencias, Universidad de Sevilla, España. \\ garcia-carmona@us.es
}

RESUMEN • Se destaca el potencial didáctico de ciertas noticias científicas de la prensa diaria para introducir la naturaleza de la ciencia (NDC) en la educación científica. A fin de ponerlo de manifiesto, se analiza interpretativamente el contenido de un repertorio de cuarenta noticias relacionadas con la Física, publicadas en periódicos españoles de gran difusión. El análisis revela la idoneidad del repertorio, tanto por la sintonía de su contenido con la visión actual de la NDC como por las posibilidades didácticas para su uso en el aula, de acuerdo con las tendencias actuales en la enseñanza de esta. Se sugiere, asimismo, su utilización en los programas de formación del profesorado, a fin de mejorar su comprensión sobre la NDC y, consecuentemente, su enseñanza.

PALABRAS CLAVE: alfabetización científica; educación científica; naturaleza de la ciencia; noticias científicas; prensa diaria.

\begin{abstract}
Newspaper reports of certain pieces of science news have the pædagogical potential of being able to introduce the Nature of Science (NOS) in science classes. This is illustrated in the present paper with an interpretative content analysis of a repertoire of 40 physics-related news items published in major Spanish newspapers. The analysis reveals that: (a) the information provided on NOS in the vast majority of the news items was quite consonant with the currently accepted concept, and (b) it suggests a diversity of pædagogical possibilities according to current trends in NOS teaching. Finally, it should be stressed that reflexive analysis on NOS using science news items in the press ought to be encouraged as a basic resource in science teacher education programs.
\end{abstract}

KEYWORDS: nature of science; newspapers; science education; scientific literacy; scientific news.

Fecha de recepción: septiembre 2013 • Aceptado: enero 2014 


\section{INTRODUCCIÓN Y PLANTEAMIENTO DE LA CUESTIÓN}

La alfabetización científica exige no solo adquirir conocimientos científicos y algunas habilidades procedimentales, así como desarrollar actitudes de interés y responsabilidad ante situaciones relacionadas con la ciencia. También demanda entender qué es la ciencia, cómo se construye y evoluciona; cómo influye su avance en el desarrollo sociocultural (y viceversa), etc. (Hodson, 2008; OCDE, 2009). Es decir, una comprensión básica de lo que se denomina naturaleza de la ciencia (NDC).

Como se recoge en informes internacionales recientes (e.g., Eurydice, 2011), la NDC forma parte de la inmensa mayoría de currículos oficiales de la ciencia escolar, sin embargo, su incidencia en las aulas es todavía escasa o manifiestamente infructuosa, a tenor de lo que revela la literatura al respecto (Lederman, 2007; García-Carmona, Vázquez y Manassero, 2012). En consecuencia, y para favorecer el afianzamiento de contenidos de NDC en la educación científica, se deben impulsar programas de formación (inicial y continua) que ayuden al profesorado a comprenderlos e integrarlos en su enseñanza (Bell, Matkins y Gansneder, 2011; García-Carmona, Vázquez y Manassero, 2011).

Pero todavía se ha avanzado poco en ello, al menos en Espańa, según estudios recientes (Guisasola y Morentin, 2007; Vázquez et al., 2013) que ponen de manifiesto la mejorable formación del profesorado de ciencia en cuestiones relacionadas con la NDC. Además, la integración de contenidos de NDC en la educación científica se ve obstaculizada, con frecuencia, por la infravaloración de estos frente a otros contenidos más clásicos del currículo, incluso entre profesorado con la formación pertinente (Akerson y Abd-El-Khalick, 2003). Por esta razón, urgen propuestas didácticas innovadoras, concretas y útiles, que orienten $-\mathrm{y}$, sobre todo, motiven- al profesorado de ciencia «de a pie» para integrar la enseñanza de nociones de NDC en sus clases.

En la elaboración de tales propuestas conviene analizar antes, entre otras cuestiones, la utilidad que ofrecen determinados recursos a los que el profesorado puede tener fácil acceso para dicho propósito. Las noticias científicas de la prensa diaria pueden ser uno de estos recursos, cuyo potencial didáctico en la enseńanza de la ciencia ha sido profusamente destacado (e.g., McClune y Jarman, 2010; Jiménez, Hernández y Lapetina, 2010; Oliveras, Márquez y Sanmartí, 2013). Sin embargo, su utilización en el caso de la enseñanza de la NDC aún no ha sido suficientemente explorada (Cakmakci y Yalaki, 2011).

Por ello, y dado el interés del autor por promover dicho recurso para enseñar la NDC en clases de Física de educación secundaria, se planteó realizar un estudio, de corte interpretativo, guiado por las siguientes cuestiones:

1. ¿Se puede seleccionar de la prensa diaria algún repertorio de noticias científicas, relacionadas con la Física, que contengan alusiones a los distintos rasgos característicos de la NDC?

2. ¿Qué potencial didáctico presentan esas noticias científicas para enseñar nociones sobre la NDC en clases de Física?

La finalidad del presente artículo es mostrar los resultados e implicaciones derivadas del análisis en torno a las preguntas anteriores.

\section{MARCO TEÓRICO Y ANTECEDENTES}

\section{Qué enseñar sobre NDC}

El concepto de NDC integra características de la ciencia emanadas de reflexiones hechas por científicos, filósofos, sociólogos, historiadores y educadores en ciencia. Esta confluencia multidisciplinar ha enriquecido el concepto, pero también ha generado un candente debate en torno a cuáles deben ser 
los rasgos que lo caracterizan y cómo estos se han de transponer didácticamente para su inclusión en la educación científica (García-Carmona, Vázquez y Manassero, 2012). Sin entrar en este debate, para dar impulso a la presencia de la NDC en el aula de ciencia, lo esencial es que el profesorado cuente con algún referente concreto e inteligible. Por ello, en los últimos años han surgido algunos consensos respecto a qué enseñar sobre NDC (Acevedo et al., 2007). Puede decirse que estos consensos comparten una buena parte de las concepciones respecto a qué es la ciencia, cómo se construye y evoluciona, qué circunstancias influyen en (o son influenciadas por) su desarrollo, etc. Y solo se diferencian en pequeńos matices en cuanto a la cantidad de aspectos considerados, o en la forma de enunciarlos.

De las propuestas de McComas y Olson (1998), Osborne et al. (2003) y Bell (2009) pueden establecerse los siguientes rasgos característicos de la NDC:

- Observación e inferencia. La ciencia es una combinación de observaciones e inferencias. La observación se refiere al uso de los cinco sentidos, ayudado con frecuencia de tecnología, para obtener información sobre fenómenos de la naturaleza. La inferencia consiste en elaborar interpretaciones o explicaciones de los fenómenos a partir de las observaciones, y normalmente integra entidades que no son directamente observables. Por eso, es posible y legítimo que los científicos hagan distintas interpretaciones de un mismo fenómeno.

- Leyes y teorías científicas. Las leyes científicas son enunciados de las relaciones entre fenómenos -expresadas a menudo en términos matemáticos- surgidas de los modelos construidos por los científicos para interpretar la realidad observada. Y las teorías científicas son explicaciones inferidas a partir de las observaciones. Son, pues, dos tipos distintos de conocimiento científico que no se convierten unos en otros, en sentido jerárquico.

- Evidencia empirica. El conocimiento científico se basa fundamentalmente en la evidencia empírica, es decir, en datos cuantitativos y cualitativos sobre los fenómenos que se analizan. Aunque algunos conceptos científicos son plenamente teóricos, en tanto que surgen de razonamientos lógicos, al final todas las ideas científicas deben ser comprobadas experimentalmente para ser consideradas válidas. No es suficiente el resultado de un solo experimento para establecer un nuevo conocimiento.

- Métodos de la ciencia. No existe un método científico universal. Los científicos emplean una amplia variedad de enfoques y estrategias para generar conocimiento científico, incluyendo observación, inferencia, experimentación... En ocasiones, se producen hallazgos casuales o inesperados que suponen también importantes avances para la ciencia.

- Creatividad. En la construcción de conocimiento científico, los científicos tienen que ser imaginativos y creativos para delimitar las preguntas que se han de investigar, establecer las mejores hipótesis explicativas y diseñar los mejores experimentos (incluyendo instrumentos de medida) que les permitan comprobar la validez de estas e interpretar los datos obtenidos.

- Objetividad y subjetividad. El conocimiento científico ya establecido, las creencias, experiencias, expectativas, intereses, etc., de los científicos influyen en la planificación y recopilación de los datos de sus nuevas observaciones, así como en su interpretación. Por eso, aunque el conocimiento científico puede ser considerado objetivo, su construcción y establecimiento requiere una aprobación consensuada de la comunidad científica; de ahí que los científicos tiendan a ser escépticos y a aplicar mecanismos de autochequeo, para mejorar la objetividad de sus conclusiones. La subjetividad proporciona, pues, la cautela necesaria para la construcción efectiva del conocimiento científico, y no puede ser (ni sería deseable que lo fuera) completamente eliminada de la actividad científica.

- Carácter provisional y evolutivo del conocimiento cientifico. El conocimiento científico es tentativo, es decir, planteado con el intento de explicar fenómenos de la naturaleza, y es el mejor 
conocimiento del que se dispone en cada momento sobre estos. Los avances en las técnicas e instrumentos de investigación empleados, así como en el conocimiento teórico, posibilitan la aparición de nuevas pruebas o reinterpretaciones de las leyes y teorías establecidas, lo que puede dar lugar a su desestimación y/o a la aceptación de otras nuevas que expliquen mejor los fenómenos. Estos cambios no son inmediatos, y vienen precedidos de cierto escepticismo y de discusiones en la comunidad científica. Pero, una vez aceptado, el nuevo conocimiento suele ser sólido y duradero. Por tanto, es razonable confiar en el conocimiento científico, aunque pueda cambiar en el futuro.

- Ciencia y sociedad. Como actividad humana, la ciencia se ve afectada por las condiciones y circunstancias del contexto sociocultural y económico en el que se desarrolla. De igual modo, la ciencia y sus avances influyen en la configuración de la sociedad y la cultura en la que se integra. Las decisiones en el desarrollo y aplicación de la ciencia no son neutrales, y, a veces, entran en conflicto con valores morales y éticos de ciertos grupos sociales.

\section{Inclusión de la NDC en el currículo de ciencia escolar}

Se plantean dos modos de incluir la NDC en el currículo de la ciencia escolar: 1) integrada con los contenidos curriculares tradicionales (e.g., tratar la provisionalidad del conocimiento científico en el contexto de la evolución de los modelos atómicos), o 2) insertada, es decir, como contenido del currículo diferenciado del resto. Las investigaciones aún son escasas para determinar cuál de ambos planteamientos es más efectivo. Akerson y Donnelly (2010) encontraron que integrando nociones sobre la NDC con el resto de contenidos se mejoran las visiones de los estudiantes sobre cuestiones como la distinción entre observación e inferencia, la creatividad, la provisionalidad, la evidencia empírica y, en menor grado, la subjetividad. Leach, Hind y Ryder (2003) lograron igualmente buenos resultados impartiendo, separadamente de los demás contenidos, lecciones específicas de NDC. Khishfe y Lederman (2007) compararon la eficacia de ambos enfoques (integración o inserción) con estudiantes de secundaria, y observaron que todos mejoraron sus visiones sobre la NDC, con independencia del enfoque. Así pues, y a falta de mayores pruebas concluyentes, lo que sí parece ser un factor determinante en el éxito de la enseñanza de la NDC, en cualquiera de los dos enfoques, es que esta sea explícita y reflexiva (Acevedo, 2009).

Por otra parte, los contextos habituales en la enseńanza de nociones sobre la NDC son los siguientes (García-Carmona, Vázquez y Manassero, 2012):

- Enseñanza de la NDC mediante análisis de problemáticas sociocientificas. Favorece que los estudiantes elaboren argumentos basados en pruebas y conocimientos científicos vigentes, intereses medioambientales o legales que interfieren con intereses económicos, etc. Cobra especial relevancia el análisis de pruebas contrarias en torno a alguna cuestión sociocientífica de controversia (cambio climático, construcción de centrales nucleares, posibles efectos nocivos de la radiación de los teléfonos móviles para la salud, etc.) porque, para decidir en quién y en qué confiar, los estudiantes han de basar sus argumentos en «indicadores de fiabilidad» a partir de la aceptación de la autoridad de investigadores y otras fuentes de información, la evaluación de fuentes de información, utilizando términos como intereses, neutralidad o competencia, entre otros.

- Enseñanza de la NDC a través de la historia de la ciencia. Constituye un escenario imprescindible para entender la NDC, pues permite, entre otros aspectos, a) ejemplificar el desarrollo de las teorías científicas; $b$ ) conocer las relaciones ciencia-sociedad existentes en cada época; $c$ ) ilustrar el carácter universal y multicultural de la ciencia, y d) contribuir a la integración y coherencia del currículo de ciencia. No obstante, al enseñar historia de la ciencia se ha de cuidar no caer 
en deformaciones o simplificaciones excesivas que den lugar a estereotipos extremos (Allchin, 2004). Es de especial interés un enfoque que permita a los estudiantes conocer los tipos de razonamientos que ayudaron a los científicos del pasado a interpretar fenómenos científicos. Asimismo, destacan los juegos rol, donde los estudiantes representan pasajes de la historia y vida de algunos científicos.

- Enseñanza de la NDC a partir de actividades experimentales. La mera realización de investigaciones científicas escolares no garantiza que se aprenda sobre NDC. Para que surta tal efecto es preciso que vayan acompañadas de tareas metarreflexivas sobre la importancia de tomar decisiones consensuadas respecto a cómo hacer las observaciones y mediciones, qué instrumentos emplear para ello, cómo organizar los datos, cómo superar las dificultades surgidas, qué criterios acordar en la obtención de conclusiones, etc. (García-Carmona, 2012). Además, la meditación sobre cuestiones relativas a la NDC en una actividad experimental no solo no supone una desviación del contenido de ciencia escolar tratado, sino que favorece la comprensión de los fenómenos y conceptos científicos implicados.

\section{Aproximación a la NDC con noticias científicas de la prensa diaria}

Además de los contextos anteriores, hay que tener presentes los recursos didácticos para la enseñanza de la NDC. Al igual que los libros de historia de la ciencia, o películas de contenido históricocientífico, el análisis de noticias científicas de la prensa diaria puede constituir un recurso idóneo para aprender sobre la NDC. No en vano, en muchos casos se informa de efemérides sobre descubrimientos y personajes científicos relevantes, si bien las noticias científicas de la prensa tienen un extra respecto a esos otros recursos: que suelen informar de acontecimientos científicos contemporáneos, poniéndose así de relieve el dinamismo permanente y en tiempo real de la ciencia.

Como avanzábamos en la introducción, el análisis de noticias científicas es, en general, un recurso didáctico ampliamente reconocido en el ámbito de la Didáctica de la Ciencia. Oliveras y Sanmartí (2009) seńalan al respecto que:

... la lectura de textos con contenido científico de diferentes fuentes tiene un papel fundamental en el aprendizaje de las ciencias, no solo para mejorar la comprensión de fenómenos científicos sino también para ayudar al alumnado a desarrollar una serie de capacidades para desenvolverse en el mundo y poder discutir con argumentos científicos y con espíritu crítico problemas de relevancia social (pp. 238-239).

Efectivamente, si la educación científica tiene entre sus finalidades que los estudiantes sigan aprendiendo (de y sobre) ciencia tras su periodo de escolarización, no se puede pasar por alto el potencial de las noticias científicas de la prensa (Hodson, 2008), no solo por su contenido científico (datos, descubrimientos, avances...), sino también por las informaciones que proporcionan sobre procesos de construcción de conocimiento científico, debates en torno a problemáticas sociocientíficas, etc., que pueden auspiciar el pensamiento crítico y reflexivo en el lector. Desde esta perspectiva, el análisis de determinadas noticias científicas puede favorecer la comprensión de ciertos rasgos de la NDC (Shibley, 2003; García-Carmona, 2013); sin embargo, el recurso se utiliza poco en la enseńanza de esta (Cakmakci y Yalaki, 2011). Además, conviene tener presente que las noticias científicas de la prensa no se escriben pensando en su uso educativo (Jarman y McClune, 2007, cit. en Jiménez, Hernández y Lapetina, 2010: 108); por tanto, es pertinente valorar antes su adaptabilidad al ámbito escolar. De ahí que se plantee en este trabajo la necesidad de analizar qué contenidos y enfoques de la NDC suelen incluirse en noticias de la prensa diaria espańola, con vistas a plantear propuestas didácticas factibles para su implementación en clase. 


\section{METODOLOGÍA}

Se analizaron cuarenta noticias relacionadas con la Física, publicadas entre los años 2006 y 2013, en algunos de los diarios españoles con mayor difusión: El Pais (18 noticias), $A B C$ (11 noticias), El Mundo (7 noticias), Público.es (2 noticias) y Eldiario.es (1 noticia) (anexo). Estas se extrajeron de una primera selección compuesta por más de cincuenta noticias sobre Física, ${ }^{1}$ tras descartar aquellas que no hacían alusión a ninguno de los rasgos definitorios de la NDC. Se obtuvo así un repertorio de noticias seleccionado por conveniencia (no se revisaron todas las noticias científicas publicadas en los mencionados diarios durante el periodo 2006-2013, ni se consultaron todos los diarios de la prensa espańola), con lo cual, los resultados y las conclusiones del análisis tienen un alcance limitado al repertorio.

$\mathrm{El}$ análisis se concretó en valorar qué atención recibe la NDC en el repertorio de noticias seleccionado, atendiendo a: $a$ ) la frecuencia con que son tratados los distintos rasgos de la NDC, $b$ ) los contextos o escenarios en los que estos se presentan y c) su distribución y diversidad según distintas temáticas de la Física. No se trató de encontrar, necesariamente, alusiones explícitas a dichos rasgos, sino de valorar, más bien, si el contenido de las noticias presentaba algún potencial en aras de promover en el aula un análisis reflexivo respecto a la NDC.

Para proveer al estudio de cierta fiabilidad se siguió una estrategia de análisis intraobservador (Padilla, 2002). Como de la mayoría de noticias podía inferirse más de un rasgo de la NDC, el análisis se repitió en dos momentos diferentes para afinar en la disección e interpretación de estos, sobre todo en los casos que suscitaron más dudas. Por otra parte, con objeto de abundar en la confirmabilidad del estudio (el equivalente al criterio de objetividad en los análisis cualitativos), se utilizaron descriptores de baja inferencia (Latorre, 2003) que, en este caso, son las citas textuales extraídas de algunas de las noticias analizadas, como se verá después.

\section{RESULTADOS}

\section{Frecuencias y alusiones a los distingos rasgos de la NDC en la muestra de noticias}

Primero se analizó la frecuencia con la que se alude a los distintos rasgos de la NDC en el repertorio de noticias (figura 1). Puede decirse que este es bastante completo, ya que incluye noticias que tocan todos los rasgos seleccionados como característicos de la NDC.

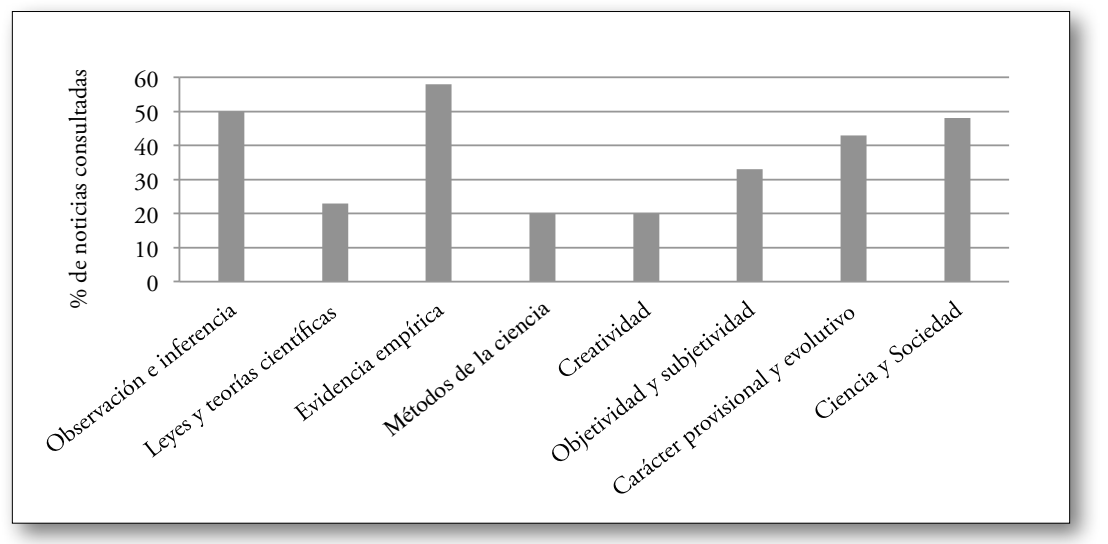

Fig. 1. Frecuencia con que se alude en el repertorio de noticias científicas a los distintos rasgos de la NDC $(\mathrm{N}=40)$.

1. Cuando la misma noticia fue publicada en más de un diario, solo se escogió la de uno de ellos. 
Los rasgos de la NDC más frecuentes son: $i$ ) la necesidad de tener evidencia empírica en la construcción del conocimiento científico (58\% de las noticias); ii) la importancia y distinción entre la observación y la inferencia en la investigación científica (50\%); iii) las influencias mutuas entre la ciencia y la sociedad en el desarrollo de ambas (48\%), y iv) el carácter provisional y evolutivo del conocimiento científico (43\%). Mientras que son menos recurrentes las noticias que se refieren a: $i)$ los métodos y circunstancias con los que se construye la ciencia, incluyendo la creatividad de los científicos (ambos rasgos, en un $20 \%$ de las noticias); ii) el surgimiento y vigencia de ciertas leyes y teorías científicas, así como sus limitaciones (23\%), y iii) la presencia e importancia de la objetividad-subjetividad en la construcción del conocimiento científico (33\%). En la tabla 1 se exponen, a modo de ejemplos, fragmentos de noticias referidos a los distintos rasgos de la NDC.

Cabe destacar, además, que la lectura de la mayoría de noticias seleccionadas suscita con relativa naturalidad una reflexión en torno a los distintos rasgos de la NDC, es decir, que estos son fácilmente identificables y, por tanto, no habría que forzar suplementariamente su abordaje en clase. Solo se observa un aspecto para el que esto no sucede tan claramente dentro del rasgo leyes y teorías cientificas: las diferencias y relaciones entre ambos tipos de conocimiento. No obstante, existen algunas noticias del repertorio propicias para plantearlo en clase, por ejemplo, el segundo fragmento de la tabla 1 , que se refiere a cómo la interpretación de ley empírica de Planck, sobre la radiación emitida por objetos según su temperatura, obligó al científico a replantearse los fundamentos de la física de la época, propiciando así el nacimiento de la teoría cuántica.

Por otra parte, puede decirse que la información dada sobre la NDC en las noticias del repertorio es, en general, adecuada. Solo se han encontrado dos casos en los que se hacen afirmaciones discordantes con la visión actual de la NDC. Uno se refiere a la idea de que la ciencia describe la realidad física tal cual es, y el otro apunta a la existencia de un único método científico:

La paradoja de Lucky Luke es que disparaba más rápido que su sombra: más deprisa que la luz que la proyectaba. Es una paradoja tremebunda, porque «la teoría de la relatividad de Einstein no es una teoría, sino una descripción comprobada y precisa de la realidad» («Más rápido que su propia sombra», El País, 2011).

La comisaria europea para la Investigación [...] subrayó la importancia del método científico, mostrando su preocupación por [...] que «se otorgue el mismo peso a las opiniones arbitrarias que a una verdad probada y verificada» («La ciencia reivindica su independencia de los mercados», El País, 2012).

Si bien las inadecuaciones anteriores no suponen más que una buena ocasión para reflexionar y comprender mejor los rasgos de la NDC implicados. Sobre esta idea, Allchin (2012) alude al análisis de los errores científicos como una excelente oportunidad para enseñar sobre NDC. 
Tabla 1.

Fragmentos de noticias que aluden o invitan

a una reflexión en torno a los rasgos característicos de la NDC

\begin{tabular}{|c|c|}
\hline $\begin{array}{c}\text { Rasgos } \\
\text { de la NDC }\end{array}$ & Fragmentos ilustrativos \\
\hline $\begin{array}{l}\text { Observación } \\
\text { e inferencia }\end{array}$ & $\begin{array}{l}\text { "... si estas partículas superasen la velocidad de la luz, los datos registrados en el detector Opera serían } \\
\text { bien distintos de lo que sus responsables han anunciado [...] unos neutrinos superlumínicos perderían } \\
\text { energía muy rápidamente y el Opera habría detectado solo los de energía inferior a un cierto límite [...] } \\
\text { "Por lo tanto, refutamos la interpretación superlumínica de los resultados de Opera", escriben Cohen } \\
\text { y Glashow en su análisis..." ("Dos reconocidos físicos refutan el experimento de los neutrinos», El País, } \\
\text { 2011). }\end{array}$ \\
\hline $\begin{array}{l}\text { Leyes y teorias } \\
\text { cientificas }\end{array}$ & $\begin{array}{l}\text { «n 1900, el físico alemán Max Planck se enfrentaba a un fenómeno que estaba en total desacuerdo con } \\
\text { la física clásica: el perfil de la gráfica de la radiación emitida por objetos a cierta temperatura. Planck pro- } \\
\text { puso una solución desesperada, pero increíblemente acertada [...] en 1905, Albert Einstein utilizó este } \\
\text { hallazgo para explicar el efecto fotoeléctrico. [...] El modelo (de Bohr), pese a sus limitaciones, explicaba } \\
\text { muchos resultados de las líneas espectrales de los gases y del orden de los elementos en la tabla periódica. } \\
\text { Hoy sabemos que el átomo de Bohr es demasiado simple, pero introduce rasgos importantes de la física } \\
\text { atómica» («El átomo cuántico cumple } 100 \text { ańos», El País, 2013). }\end{array}$ \\
\hline $\begin{array}{l}\text { Evidencia } \\
\text { empirica }\end{array}$ & $\begin{array}{l}\text { "Partamos de la base de que la teoría de la relatividad especial de Einstein es, al menos, aproximada- } \\
\text { mente correcta, y si tiene un fallo debe ser pequeño, porque hemos hecho muchas pruebas, en muchas } \\
\text { direcciones, y resulta siempre correcta. [...] otros experimentos diferentes han observado millones de } \\
\text { interacciones de neutrinos en los que debería haber aparecido esa emisión de pares electrón-positrón, } \\
\text { y no aparecen» («Si este experimento es correcto le diré a la naturaleza: vale, me rindo», El País, 2011). }\end{array}$ \\
\hline $\begin{array}{l}\text { Métodos } \\
\text { de la ciencia }\end{array}$ & $\begin{array}{l}\text { «Producir ciencia y aplicarla no es tarea fácil. Se requieren notables dosis de profesionalidad, capacidad } \\
\text { para hacerse preguntas relevantes, formación técnica y dotes analíticas para ofrecer respuestas contrasta- } \\
\text { bles, tanto en las primeras fases de la actividad científica [...] como cuando se da cuenta de los resultados } \\
\text { por medio de la publicación en revistas especializadas. La dinámica tradicional consiste en la formulación } \\
\text { de preguntas, emanadas de hipótesis, teorías, o cúmulo de datos, que suscitan interrogantes y reclaman } \\
\text { explicaciones. La respuesta se da mediante la experimentación con diseńos adecuados y la utilización de } \\
\text { las mejores metodologías existentes» («Cualificación e independencia», El País, 2013). }\end{array}$ \\
\hline Creatividad & $\begin{array}{l}\text { "Cuando Pierre tenía en la cabeza un experimento, imaginaba el aparato de medida que le hacía falta, } \\
\text { y él era capaz de hacerlo" [...]. "Era un verdadero manitas". Su mujer Marie aprovechó esa capacidad de } \\
\text { su marido y consiguió sacar un provecho inimaginable a esas herramientas» ("Marie Curie, la observadora de } \\
\text { lo invisible», ElMundo, 2013). }\end{array}$ \\
\hline $\begin{array}{l}\text { Objetividad } \\
\text { y subjetividad }\end{array}$ & $\begin{array}{l}\text { "... la comunidad científica internacional [es] quien los juzga [resultados de la actividad científica] de } \\
\text { acuerdo con un sistema de evaluación basado en la autonomía y el escepticismo }[\ldots] \text { sometiendo los }[\ldots] \\
\text { artículos al juicio de expertos de la máxima cualificación, independencia, generosidad y sin conflictos } \\
\text { de intereses }[\ldots] \text { Es lo que se llama }[\ldots] \text { evaluación por pares. }[\ldots] \text { Como los productos de la actividad } \\
\text { científica nunca son incuestionables, siempre hay varios evaluadores y son los coordinadores }[\ldots] \text { quie- } \\
\text { nes zanjan las diferencias. Las verdades científicas no son dogmas sino verdades revisables }[\ldots] \text { El avance } \\
\text { científico se logra por consenso [...] Entre los elementos de la negociación se dan las controversias» } \\
\text { ("Cualificación e independencia», El País, 2013). }\end{array}$ \\
\hline $\begin{array}{l}\text { Carácter } \\
\text { provisional } \\
\text { y evolutivo } \\
\text { del conocimien- } \\
\text { to cientifico }\end{array}$ & $\begin{array}{l}\text { «Plutón ha dejado de ser considerado un planeta [...] A esta conclusión han llegado los casi } 2.500 \text { cientí- } \\
\text { ficos participantes en la reunión de la Unión Astronómica Internacional [...] llevan dos años de intensos } \\
\text { debates para acordar una definición, después de que Brown descubriese en } 2003 \text { a UBS313 [...], lo cual } \\
\text { planteó el problema de si debía ser reconocido o no como planeta, dado que es más grande que Plutón } \\
{[\ldots] \text { La definición de planeta aprobada por unanimidad [...] viene precedida de } 2 \text { años de debates y } 10} \\
\text { días de controvertidas sesiones...» («Plutón deja de ser considerado planeta tras el acuerdo de la comu- } \\
\text { nidad astronómica internacional», El Mundo, 2006). }\end{array}$ \\
\hline $\begin{array}{l}\text { Ciencia } \\
y \text { sociedad }\end{array}$ & $\begin{array}{l}\text { «... Los científicos siempre serán vulnerables a personas ignorantes o miopes en el poder }[\ldots] \text { cuanto ma- } \\
\text { yor sea la transparencia y el poder de las redes sociales, será más fácil detectar malas políticas y resistirse } \\
\text { a ellas }[. . .] \text {. Otras veces, las resistencias a los avances científicos se producen por motivos morales» («La } \\
\text { austeridad hará que se resienta la capacidad de conocimiento de todos los países occidentales», } A B C, 2013 \text { ). }\end{array}$ \\
\hline
\end{tabular}


Asimismo, se estimó oportuno conocer qué cantidad de rasgos de la NDC eran incluidos en una misma noticia. Porque, ante programas curriculares habitualmente sobrecargados, es posible que este recurso tenga mayor acogida entre el profesorado si permite abordar distintos rasgos de la NDC con el menor número de noticias.

El máximo número de rasgos de la NDC, insinuados en una misma noticia del repertorio, fue 6 y correspondió a «Lo que Einstein llamó su mayor error sería mi mayor hallazgo» (El Pais, 2013), del cual se exponen fragmentos con alusiones a estos en la tabla 2 . Asimismo, es destacable que el $56 \%$ de las noticias alude a entre 3 y 6 rasgos distintos de la NDC. De modo que, a la hora de elegir noticias que traten diversos rasgos, el repertorio es también bastante sugerente.

Tabla 2.

Fragmentos de la noticia «Lo que Einstein llamó su mayor error sería mi mayor hallazgo» (El País, 2013) con alusiones a diversos rasgos de la NDC

\begin{tabular}{|c|c|}
\hline $\begin{array}{c}\text { Rasgos } \\
\text { de la NDC }\end{array}$ & Fragmentos de la noticia que aluden a estos \\
\hline $\begin{array}{l}\text { Observación } \\
\text { e inferencial } \\
\text { evidencia } \\
\text { empirica }\end{array}$ & $\begin{array}{l}\text { «Todo empezó }[\ldots] \text { con unas observaciones de galaxias cuyos resultados eran tan locos que, cuando los } \\
\text { vio, se preguntó: “¿Qué hemos hecho mal?”. Temió incluso que aquello fuera el final de su carrera de } \\
\text { científico }[\ldots] \text { Repasó todo de nuevo }[\ldots] \text { con su equipo }[\ldots] \text { y seguía saliendo lo mismo: la expansión del } \\
\text { universo, en lugar de estar frenándose, estaba acelerándose. Pero decían lo mismo los resultados idénticos } \\
\text { del equipo competidor estadounidense }[\ldots] \text { "Entonces me di cuenta de que iba a ser algo grande”...». }\end{array}$ \\
\hline $\begin{array}{l}\text { Objetividad } \\
\text { y subjetividadl } \\
\text { carácter } \\
\text { provisional } \\
\text { y evolutivo }\end{array}$ & $\begin{array}{l}\text { "... muchos científicos }[\ldots] \text { recibieron la noticia con incredulidad. "Es que la ciencia es conservadora: } \\
\text { estamos siempre poniendo a prueba nuestras ideas, pero cuando una pone todo patas arriba, somos } \\
\text { escépticos"...». }\end{array}$ \\
\hline $\begin{array}{l}\text { Leyes y teorias } \\
\text { cientificas/ } \\
\text { observación } \\
\text { e inferencia }\end{array}$ & $\begin{array}{l}\text { «.. las galaxias están separándose unas de otras y cuanto más lejos están del observador, mayor es la velo- } \\
\text { cidad aparente a la que se alejan de él. [...] Einstein no lo esperaba. Creía, como todos los científicos en- } \\
\text { tonces, que el universo era estático y, como sus ecuaciones de la relatividad le daban un cosmos que colap- } \\
\text { saría sobre sí mismo, había introducido la constante cosmológica para frenarlo. Cuando Hubble constató } \\
\text { que el universo estaba en expansión, ya no hacía falta en las ecuaciones de Einstein ese aparente artificio } \\
\text { [...] Y resulta que ahora es la mejor teoría que los físicos tienen para explicar su gran descubrimiento». }\end{array}$ \\
\hline $\begin{array}{l}\text { Ciencia } \\
y \text { sociedad }\end{array}$ & $\begin{array}{l}\text { "... es necesario invertir esfuerzo en la investigación fundamental. "La ciencia, la astronomía, es pensar, } \\
\text { y al hacerlo abrimos la mente a nuevas ideas, a cómo abordar problemas [...], y esto es rentable para la } \\
\text { sociedad" [...] "La web nació en el [...] CERN, un centro de investigación básica; y el wif lo inventó } \\
{[\ldots . \text { John O’Sullivan, que estudiaba cómo se evapora un agujero negro y tenía que solucionar el problema }} \\
\text { de cómo rebotan las ondas radio en la galaxia [...] la solución }[\ldots . .] \text { se tradujo en el wifi"». }\end{array}$ \\
\hline
\end{tabular}

En consecuencia, puede decirse que el repertorio de noticias, en general, incluye una información variada y explícita que ayudaría a adquirir una visión adecuada de la ciencia, esto es, un campo de conocimientos tentativos donde no existen ideas privilegiadas, que son objeto de posible revisión, o incluso rechazo, a la luz de nuevas evidencias o reinterpretaciones de las existentes (Bell, 2009). Igualmente favorece una visión de la ciencia como elemento básico de la sociedad de cada época.

\section{Contextos en los que se desarrollan los rasgos de la NDC en las noticias}

También se clasificaron las noticias según el contexto o la perspectiva en la que se desarrolla su contenido. Se dijo en el marco teórico que los escenarios más frecuentes en la enseñanza de la NDC son el análisis reflexivo en torno a problemáticas sociocientíficas, pasajes de historia de la ciencia y el diseño y la ejecución de actividades experimentales. Sin embargo, dadas las peculiaridades de las noticias cientí- 
ficas, no tenía mucho sentido considerar este último y se decidió agruparlas finalmente en los siguientes contextos no excluyentes entre sí: $a$ ) avances y límites actuales de la ciencia, $b$ ) historia de la ciencia y c) problemáticas sociocientíficas. La figura 2 recoge la distribución de noticias según estos contextos.

Las noticias sobre los avances y límites actuales de la física son las más frecuentes $(55 \%$ de las noticias); algo de sumo interés didáctico con vistas a poner de relieve el carácter dinámico, revisable e inconcluso del conocimiento científico. También, aunque con menor frecuencia, es destacable la atención del repertorio a la historia de la ciencia (25\%) y a problemáticas sociocientíficas (35\%), dadas las bondades didácticas que ambos escenarios tienen en la comprensión de la NDC, de acuerdo con lo descrito en el marco teórico. De modo que el repertorio es didácticamente idóneo con respecto a la diversidad de contextos o perspectivas desde las que se puede analizar la NDC.

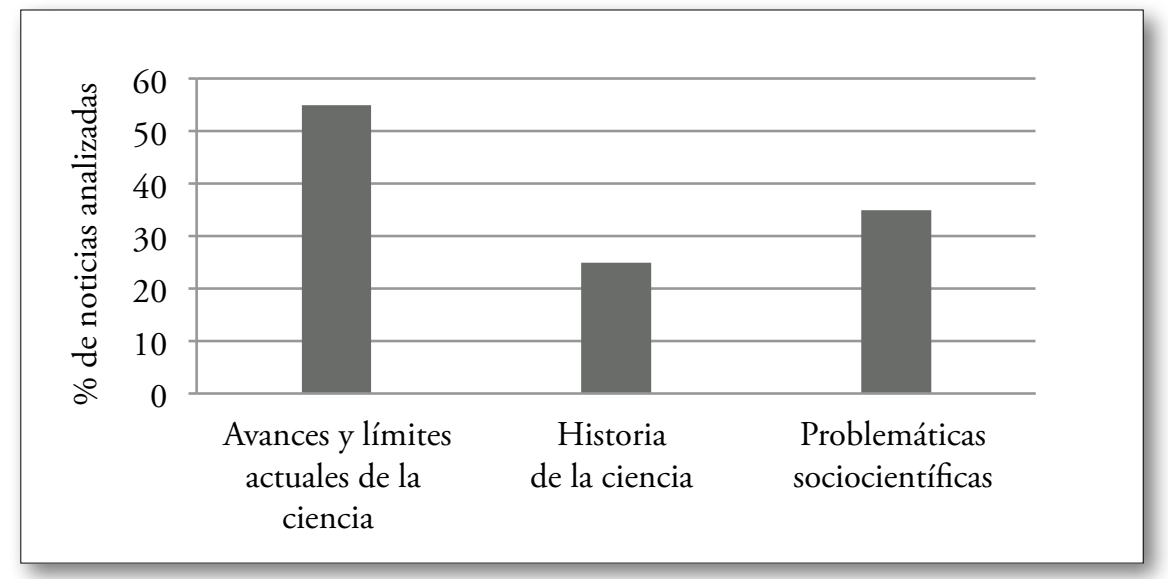

Fig. 2. Distribución de la muestra de noticias según el contexto del contenido (avances y límites actuales de la ciencia, historia de la ciencia y problemáticas sociocientíficas) $(\mathrm{N}=40)$.

\section{Frecuencia y diversidad de rasgos de la NDC en las noticias, según las temáticas de la física}

Finalmente, se catalogaron las noticias según los distintos temas o campos de investigación de la Física, con idea de favorecer la elección de unas u otras noticias a la hora de integrar la NDC en los contenidos habituales de la Física escolar.

La muestra abarca una diversidad de áreas o temas que, en algunos casos, aparecen imbricados dentro de una misma noticia. Asimismo, incluye noticias que abordan temas genéricos o transversales de la ciencia, sin ubicarse en un campo específico de esta. Ante todo ello se decidió clasificar las noticias tratando de sintonizar, por un lado, con las denominaciones predominantes en Física actualmente, y, por otro, con la estructura usual de los contenidos de la Física escolar. ${ }^{2}$ La clasificación final fue: Astronomía/Cosmología, Cambio climático, Física cuántica, Física de la Tierra, Física de partículas, Radiactividad, Relatividad y Temas transversales de la Ciencia. ${ }^{3}$

La tabla 3 sintetiza la distribución de noticias según las temáticas que tratan, los contextos o las perspectivas del contenido y los rasgos de la NDC a que aluden. Destaca que más de la mitad de las noticias se refieren a Astronomía/Cosmología (35\%), Relatividad (18\%) y Física cuántica (10\%), y

2. Si bien la muestra de noticias se recopiló pensando en su implementación con estudiantes de final de la ESO y bachillerato, lógicamente puede ser utilizada con estudiantes universitarios e incluso en procesos de formación de profesorado de ciencia.

3. Aunque el cambio climático podría integrarse en el bloque de Física de la Tierra, se optó por independizarlo dada su enjundia en la actualidad. Algo similar sucede con algunos de los restantes bloques de la clasificación. 
que las relacionadas con cada una de estas permiten abordar en clase todos los rasgos de la NDC considerados. Las noticias sobre Física de partículas (10\%) cubren 6 de los 8 rasgos de la NDC y, en muchos casos, pueden integrarse con las de Astronomía/Cosmología y Física Cuántica, lo que ampliaría la propuesta sobre estos últimos temas.

Las noticias sobre cuestiones transversales de la ciencia acaparan el 23\% del repertorio, con alusiones a 6 de los 8 rasgos de la NDC. Estas presentan, en principio, la ventaja de que pueden ser utilizadas en clase con independencia del contenido de Física objeto de enseñanza. Por lo demás, aunque las noticias menos frecuentes versan sobre Física de la Tierra y Cambio climático -con 2 noticias cada tema- y Radiactividad - una noticia-, tienen el interés de que insinúan una cantidad considerable de rasgos de la NDC: 6 y 5, respectivamente.

En cuanto a los contextos en los que se desarrollan los temas, el grupo correspondiente a Astronomía/Cosmología es el más completo al incluir noticias con alusiones a los avances y límites actuales de la ciencia, a la historia de la ciencia y a problemáticas sociocientíficas. El resto de bloques de noticias integran una o dos perspectivas, como se observa en la tabla 3.

\section{CONCLUSIONES}

Hoy ya no se cuestiona que una adecuada alfabetización científica debe integrar un conocimiento básico sobre la NDC, y es difícil encontrar una propuesta oficial de ciencia escolar que no incluya en sus metas educativas alguna alusión a ello. Sin embargo, su enseñanza no termina de ser considerada ni efectivamente desarrollada en las clases de ciencia. De ahí que la comprensión de la NDC sea, actualmente, uno de los principales retos en la educación científica.

De los distintos aspectos que se deben considerar en el impulso de la enseñanza de la NDC, se ha destacado la promoción de recursos didácticos que sean accesibles, inteligibles y fácilmente integrables por el profesorado en su programa de enseñanza. En este sentido, se ha argumentado sobre la utilización de noticias científicas de la prensa diaria, cuya lectura reflexiva, y pertinentemente guiada por el profesorado, puede favorecer la adquisición de una visión adecuada de la ciencia. Pero esto continúa siendo una hipótesis que hay que contrastar con investigación didáctica (cuestión sobre la que se encuentra trabajando el autor), porque la evidencia empírica que pueda ratificarlo es todavía escasa (Cakmakci y Yalaki, 2011).

Mientras tanto, parece pertinente profundizar en las potencialidades didácticas del propio recurso, con vistas a su uso en el aula. Surge así el presente estudio, cuyo propósito ha sido mostrar la posibilidad de seleccionar un repertorio de noticias científicas, extraídas de la prensa diaria, que resulte útil para introducir nociones sobre la NDC en clases de Física. Además, con la aspiración de que sea fácilmente accesible para el profesorado interesado en el tema, ya que todas las noticias analizadas son de libre acceso on-line (basta poner el título de la noticia en un buscador de internet).

Contestando a las cuestiones de investigación planteadas, y de acuerdo con los resultados del análisis efectuado, puede concluirse que:

- El repertorio de noticias constituye, en su conjunto, una fuente de información bastante rica y completa, en tanto que se detectan alusiones a todos y cada uno de los rasgos característicos de la NDC considerados. Además, se conjugan noticias de la actualidad científica con rememoraciones de hitos científicos del pasado, lo que favorece la idea de progreso y dinamismo continuo de la ciencia.

- Los distintos rasgos de la NDC seleccionados son fácilmente identificables en la mayoría de noticias, facilitando así una reflexión explícita en torno a estos tras su lectura. Incluso cuando 


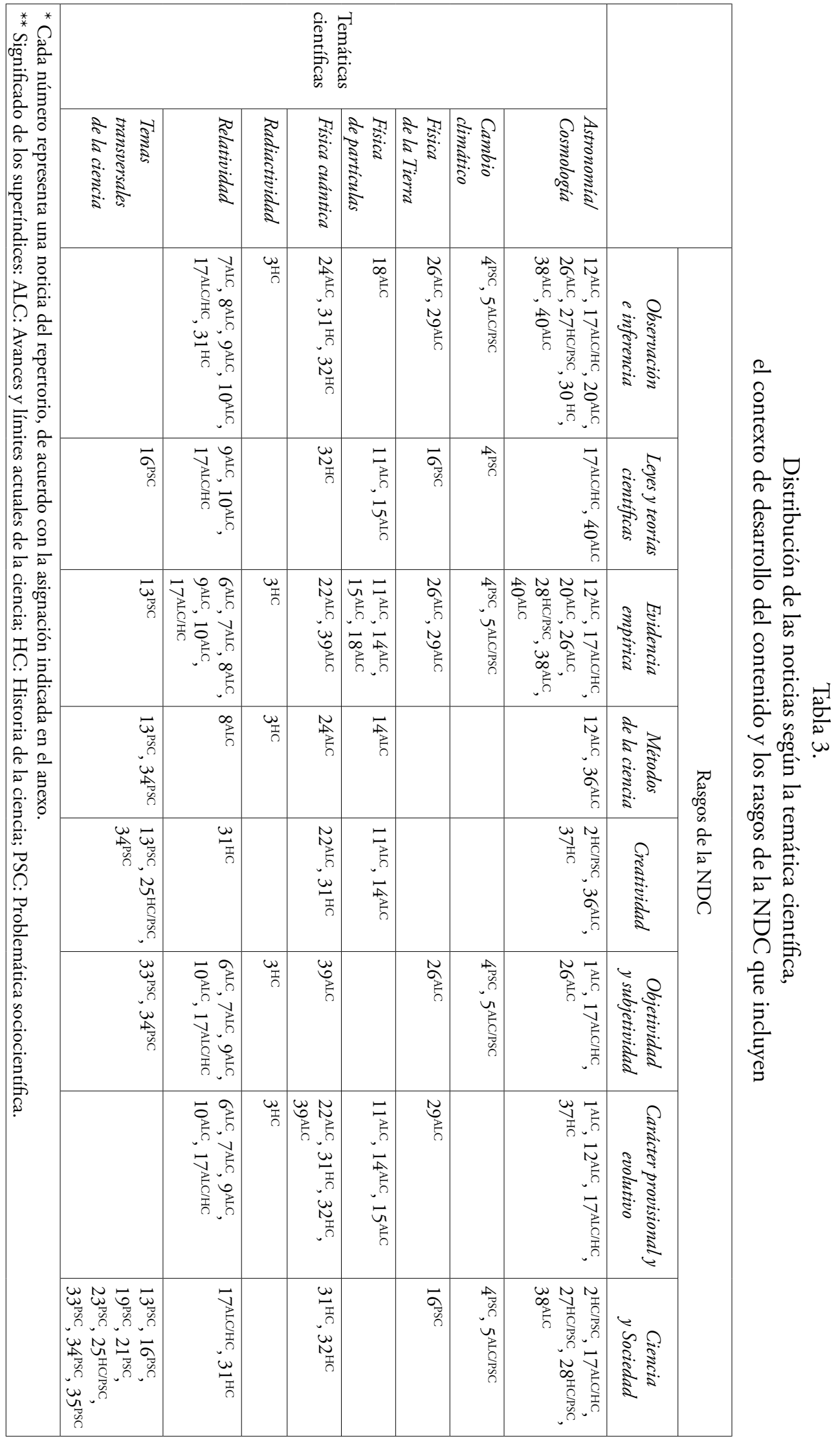


no es así, la amplitud y variedad de noticias del repertorio ofrece múltiples posibilidades para una reflexión armónica en el contexto de alguna de ellas.

- Una porción importante de noticias hace alusión a diversos rasgos de la NDC en un mismo contexto informativo, lo que favorece, por un lado, una idea integrada sobre qué es la ciencia, y, por otro, la posibilidad de abordar en clase más de un rasgo de la NDC con la menor cantidad de noticias posible.

- La información proporcionada sobre la NDC, en la inmensa mayoría de noticias científicas, es bastante consonante con la concepción que se tiene de esta actualmente. Solo en dos noticias del repertorio se ha detectado que algún rasgo no era adecuadamente tratado. Si bien ello supone igualmente una excelente ocasión para reflexionar y, por tanto, aprender sobre estos; por ejemplo, mediante la generación de conflictos cognitivos, a lo que el propio repertorio puede contribuir con otras noticias que tratan dichos rasgos de manera adecuada.

- Atendiendo a la idoneidad de disponer de distintos contextos o escenarios a través de los que introducir en clase cuestiones relativas a la NDC, el repertorio también responde a ello con cierta variedad. Las noticias se han podido clasificar en tres grupos diferentes, según atendían a avances y límites actuales de la ciencia, a la historia de la ciencia o a problemáticas sociocientíficas. Ciertamente es una clasificación entre tantas otras posibles, pero puede resultar útil a la hora de seleccionar noticias conforme a los intereses didácticos de cada momento del desarrollo curricular.

- El repertorio de noticias propicia la inclusión de la NDC en el currículo desde los dos enfoques: a) de manera integrada con el resto de contenidos de la Física en la escuela, porque los distintos rasgos de la NDC se evidencian a través de diversas temáticas y líneas de investigación de la Física, como Astronomía/Cosmología, Cambio climático, Física cuántica, Física de la Tierra, Física de partículas, Radiactividad y Relatividad; y $(b)$ de manera insertada o como contenido independiente del resto, por ejemplo, con las noticias denominadas temas transversales de ciencia que, aun teniendo relación con la Física, se refieren a cuestiones genéricas de la ciencia y los científicos en general. Algo que conecta con lo recogido en los bloques de contenidos comunes de los actuales currículos españoles de ciencia para la ESO y Bachillerato.

Con todo, puede decirse que repertorios de noticias científicas como el analizado presentan un estimable potencial didáctico para abordar la NDC en clase de Física. Incluso mayor que los actuales libros de texto de ciencias, que suelen mostrar una imagen distorsionada y poco adecuada de la ciencia (Solaz-Portolés, 2010). Consecuentemente, se espera que esto anime al profesorado a utilizarlo directamente en sus clases, o bien a elaborar uno propio con otras noticias que se amolden a sus intereses docentes. Cabe decir al respecto que, si bien el repertorio se ha circunscrito al ámbito de la Física, contiene multitud de noticias que pueden ser utilizadas en la enseńanza de otras ciencias; principalmente, aquellas referidas a temas transversales de la ciencia, o al cambio climático, por citar algunas.

Respecto a la implementación de la enseñanza de la NDC con noticias científicas, las posibilidades son múltiples (una propuesta concreta y detallada, al respecto, se describe en García-Carmona [2013]); aunque es cierto que el hecho de que el profesorado posea una formación básica sobre la NDC le ayudará a hacer un uso más efectivo de estas. Por este motivo, el análisis reflexivo sobre la NDC utilizando noticias científicas de la prensa debería fomentarse también como un recurso básico en los programas de formación (inicial y permanente) del profesorado de ciencia. Pero se puede añadir un argumento más en tal sentido. El marco educativo actual, basado en el desarrollo de competencias, contempla entre sus finalidades adquirir la capacidad de aprender a lo largo de toda la vida. Y para una gran parte de la ciudadanía, tanto en etapa escolar como egresada del sistema educativo, las noticias científicas de la prensa diaria constituyen una de las principales fuentes de información sobre los avances y pro- 
blemáticas actuales de la ciencia (Hodson, 2008). Por consiguiente, parece lógico que el profesorado de ciencia deba estar preparado para enseñar a analizarlas crítica y reflexivamente desde la educación científica básica.

\section{REFERENCIAS BIBLIOGRÁFICAS}

Acevedo, J.A. (2009). Enfoques explícitos versus implícitos en la enseñanza de la naturaleza de la ciencia. Revista Eureka sobre Enseñanza y Divulgación de las Ciencias, 6(3), pp. 355-386.

Acevedo, J.A.; VÁzquez, A.; Manassero, M.A. y Acevedo, P. (2007). Consensos sobre la naturaleza de la ciencia: fundamentos de una investigación empírica. Revista Eureka sobre Enseñanza y Divulgación de las Ciencias, 4 (1), pp. 42-66.

Akerson, V.L. y AbD-El-Khalick, F. (2003). Teaching elements of nature of science: a yearlong case study of a fourth-grade teacher. Journal of Research in Science Teaching, 40 (10), pp. 1025-1049. http://dx.doi.org/10.1002/tea.10119

Akerson, V. y Donnelly, L.A. (2010). Teaching Nature of Science to K-2 Students: What understandings can they attain? International Journal of Science Education, 32 (1), pp. 97-124. http://dx.doi.org/10.1080/09500690902717283

Allchin, D. (2004). Pseudohistory and Pseudoscience. Science \& Education, 13, pp. 179-195. http://dx.doi.org/10.1023/B:SCED.0000025563.35883.e9

Allchin, D. (2012). Teaching the nature of science through scientific errors. Science Education, 96 (5), pp. 904-926. http://dx.doi.org/10.1002/sce.21019

Bell, R.L. (2009). Teaching the nature of science: Three critical questions. En Best Practices in Science Education. Carmel, CA: National Geographic School Publishing. http://dx.doi.org/10.1002/tea.20402

Bell, R.L.; Matkins, J.J. y Gansneder, B.M. (2011). Impacts of Contextual and Explicit Instruction on Preservice Elementary Teachers' Understandings of the Nature of Science. Journal of Research in Science Teaching, 48 (4), pp. 414-436. http://dx.doi.org/10.1038/458559a

Cakmakci, G. y Yalaki, Y. (2011). Popular media as a tool for teaching science and its nature. En Promoting Student Teachers' Ideas about Nature of Science through Popular Media. Hacettepe University: Science-Teacher Education Advanced Methods (S-TEAM) project.

Eurydice (2011). Science Education in Europe: National Policies, Practices and Research. Disponible en línea: http://eacea.ec.europa.eu/education/eurydice.

García-Carmona, A. (2012). Cómo enseñar Naturaleza de la Ciencia (NDC) a través de experiencias escolares de investigación científica. Alambique, 72, pp. 55-63.

García-Carmona, A. (2013). Aprender sobre la Naturaleza de la Ciencia con noticias científicas de actualidad: el caso del experimento OPERA. Alambique, 75, pp. 65-75.

García-Carmona, A.; Vázquez, A. y Manassero, M.A. (2011). Estado actual y perspectivas de la enseñanza de la naturaleza de la ciencia: una revisión de las creencias y obstáculos del profesorado. Enseñanza de las Ciencias, 29 (3), pp. 403-412.

García-Carmona, A.; Vázquez, A. y Manassero, M.A. (2012). Comprensión de los estudiantes sobre Naturaleza de la Ciencia: análisis del estado actual de la cuestión y perspectivas. Enseñanza de las Ciencias, 30 (1), pp. 23-34.

Guisasola, J. y Morentin, M. (2007). ¿Comprenden la naturaleza de la ciencia los futuros maestros y maestras de Educación Primaria? Revista Electrónica de Enseñanza de las Ciencias, 6 (2), pp. 246-262. 
Hodson, D. (2008). Towards scientific literacy. Rotterdam: Sense Publishers.

Jiménez-Liso, M.R.; Hernández-Villalobos, L. y Lapetina, J. (2010). Dificultades y propuestas para utilizar las noticias científicas de la prensa en el aula de ciencias. Revista Eureka sobre Enseñanza y Divulgación de las Ciencias, 7 (1), pp. 107-126.

Khishfe, R. y Lederman, N. (2007). Relationship between Instructional Context and Views of Nature of Science. International Journal of Science Education, 29 (8), pp. 939-961. http://dx.doi.org/10.1080/09500690601110947

Latorre, A. (2003). La investigación-acción. Conocer y cambiar la práctica educativa. Barcelona: Graó.

LEACH, J.; Hind, A. y Ryder, J. (2003). Designing and evaluating short teaching interventions about the epistemology of science in high school classrooms. Science Education, 87 (6), pp. 832-848. http://dx.doi.org/10.1002/sce.10072

Lederman, N.G. (2007). Nature of science: past, present, and future. En S.K. Abell y N.G. Lederman (eds.). Handbook of Research on Science Education. Mahwah, NJ: Lawrence Erlbaum Associates, pp. 831-879.

http://dx.doi.org/10.1002/sce.10072

Mcclune, B. y Jarman, R. (2010). Critical Reading of Science区 Based News Reports: Establishing a knowledge, skills and attitudes framework. International Journal of Science Education, 32 (6), pp. 727-752.

http://dx.doi.org/10.1080/09500690902777402

Mccomas, W.F. y Olson, J.K. (1998). The nature of science in international science education standards documents. En W.F. McComas (ed.). The Nature of Science in Science Education. Rationales and Strategies. Dordrecht: Kluwer Academic Publishers, pp. 41-52.

OCDE (2009). PISA 2009. Assessment framework-key competencies in reading, mathematics and science. París: Autor.

Oliveras, B.; Márquez, C. y Sanmartí, N. (2013). The use of newspaper articles as a tool to develop critical thinking in science classes. International Journal of Science Education, 35 (6), pp. 885-905.

Oliveras, B. y Sanmartí, N. (2009). La lectura como medio para desarrollar el pensamiento crítico. Educación Química, número extra, pp. 233-245.

http://dx.doi.org/10.1080/09500693.2011.586736

Osborne, J.F.; Collins, S.; Ratcliffe, M.; Millar, R. y Duschl, R. (2003). What «Ideas-aboutScience» should be taught in school science? A delphi study of the expert community. Journal of Research in Science Teaching, 40 (7), pp. 692-720.

http://dx.doi.org/10.1002/tea.10105

Padilla, M.T. (2002). Técnicas e Instrumentos para el Diagnóstico y la Evaluación Educativa. Madrid: CCS.

Shibley, I.A. (2003). Using newspapers to examine the nature of science. Science \& Education, 12 (7), pp. 691-702.

http://dx.doi.org/10.1023/A:1025687424931

Solaz-PortolÉs, J.J. (2010). La naturaleza de la ciencia y los libros de texto de ciencias: una revisión. Educación XX1, 13 (1), pp. 65-80.

http://dx.doi.org/10.5944/educxx1.13.1.277

Vázquez, A.; García-Carmona, A.; Manassero, M.A. y Bennàssar, A. (2013). Science Teachers' Thinking about the Nature of Science: A New Methodological Approach to its Assessment. Research in Science Education, 43 (2), pp. 781-808.

http://dx.doi.org/10.1007/s11165-012-9291-4 


\section{ANEXO \\ Relación de noticias científicas analizadas}

1. «Plutón deja de ser considerado planeta tras el acuerdo de la comunidad astronómica internacional» (El Mundo, 2006)

2. «El hereje Galileo retoma Florencia» (El País, 2009)

3. «Victor Hess contra los rayos cósmicos siderales» (El País, 2009)

4. «Klaus Hasselmann: "Los científicos del cambio climático no somos tramposos"» (El País, 2010)

5. «Es ridículo dudar de que la Tierra se calienta; ningún científico lo hace» (El País, 2010)

6. «Tensión y desacuerdo entre los físicos del experimento de los neutrinos» (El País, 2011)

7. «Dos reconocidos físicos refutan el experimento de los neutrinos» (El País, 2011)

8. «Nuevos datos, aunque no definitivos, apoyan el experimento de los neutrinos» (El País, 2011)

9. «Si este experimento es correcto le diré a la naturaleza: vale, me rindo» (El País, 2011)

10. "Más rápido que su sombra» (El País, 2011)

11. «El bosón de Higgs (¡la partícula de Diosi) en 9 claves» (Público.es, 2012)

12. «Los misterios que el bosón de Higgs aún no puede explicar» $(A B C, 2012)$

13. «La ciencia reivindica su independencia de los mercados» (Público.es, 2012)

14. «Stephen Hawking perdió una apuesta sobre la existencia del bosón de Higgs» $(A B C, 2012)$

15. “¿Hallado un agujero en el modelo estándar de la Física?» $(A B C, 2012)$

16. «El delicado papel de la ciencia en las catástrofes» (El País, 2012)

17. "Lo que Einstein llamó su mayor error sería mi mayor hallazgo» (El País, 2013)

18. «Si te preguntas el porqué de las cosas acabas en la Física» (El País, 2013)

19. "Ciencia y Capital» (El País, 2013)

20. «El $70 \%$ del universo había pasado desapercibido hasta ahora» (El País, 2013)

21. "Los trabajadores de la ciencia» (Eldiario.es, 2013)

22. «El primer átomo con forma de pera» $(A B C, 2013)$

23. «La austeridad hará que se resienta la capacidad de conocimiento de todos los países occidentales» $(A B C, 2013)$

24. «Logran observar átomos sin destruirlos por primera vez» $(A B C, 2013)$

25. «Marie Curie, la observadora de lo invisible» (El Mundo, 2013)

26. "El hallazgo del meteorito de Tunguska "es ridículo"» $(A B C, 2013)$

27. «El primer mártir de la ciencia» (El Mundo, 2013)

28. «El hereje que adivinó otros mundos» $(A B C, 2013)$

29. «El centro de la Tierra gira a distinta velocidad» $(A B C, 2013)$

30. «El telescopio de Galileo y el declive del Imperio español» (El Mundo, 2013)

31. "¿Por qué le debemos tanto a Einstein?» (El Mundo, 2013)

32. «El átomo cuántico cumple 100 años» (El País, 2013)

33. «¿Quién es capaz de poner nota a la Ciencia?» (El País, 2013)

34. "Cualificación e independencia» (El Pais, 2013)

35. «Mira, mamá! ¡Ahora sin Ciencia!» (Eldiario.es, 2013)

36. «Hay otro planeta azul gracias al silicato de su atmósfera» (El País, 2013)

37. «El príncipe de las mareas» (El Mundo, 2013)

38. «La nada creativa» (El Mundo, 2013)

39. «Un nuevo reloj atómico podría llevar a la redefinición del segundo» $(A B C, 2013)$

40. «Descubren que el Sistema Solar tiene cola, como los cometas» $(A B C, 2013)$ 


\title{
The nature of science in press articles about scien- ce: content analysis and pædagogical potential
}

\author{
Antonio García-Carmona \\ Departamento de Didáctica de las Ciencias, Universidad de Sevilla, España. \\ garcia-carmona@us.es
}

Newspaper reports of certain pieces of science news have the pædagogical potential of being able to introduce the Nature of Science (NOS) in science classes. This is illustrated in the present paper with an interpretative content analysis of a repertoire of 40 physics-related news items published in major Spanish newspapers.

The conclusions drawn from the analysis were that:

- The news repertoire studied constituted overall a rich and complete source of information since references to all the characteristic features of NOS considered were detected. In addition, recent advances in science were combined with background recollections of past scientific achievements, thus presenting an idea of the progress and continuing dynamism of Science.

- The features of NOS selected were readily identifiable in most of the items, facilitating explicit reflection on them after reading the item. Even though this was not always the case, the extent and variety of the news repertoire offers multiple possibilities for harmonious reflection on the different contexts dealt with.

- A major portion of the items referred to various features of NOS in the same single piece of science information. This not only fosters an integrated idea about what Science is, but also makes it possible to address more than one feature of NOS in class using as little information from the papers as possible.

- In the vast majority of the news items, the information provided on NOS was quite consonant with the currently accepted concept. Only in two items of the repertoire was some feature detected that was not dealt with properly. Nonetheless, this itself could provide an excellent opportunity for reflection, and therefore learning, about those features. For example, contrasting them with other pieces of science news in which they are presented adequately can help learning by generating cognitive conflicts.

- With respect to the appropriateness of having different contexts or scenarios through which to present NOSrelated questions in class, the repertoire responded to this facet with a fair variety in its subject matter. It was possible to classify the news into three groups, according to whether they dealt with current progress and boundaries in Science, with the history of Science, or with socio-scientific problematic issues. Clearly, this classification was just one among many possible, but it may be useful in helping select news items according to the teaching interests corresponding to any given stage in doing the curriculum.

- The repertoire can facilitate the inclusion of NOS in the curriculum in two ways: (i) by integrating it into the rest of the content of school physics, because the various features of NOS are illustrated in different contexts of physics research such as astronomy/cosmology, climate change, quantum physics, physics of the Earth, particle physics, radioactivity, and relativity; and (ii) by inserting it as separate content, for example, with science news items that deal with cross-cutting themes which, despite having some relation to physics, refer to generic issues of science and scientists in general, something that connects with what, in Spain's current Science curricula for the compulsory secondary education and pre-university levels, are called "blocks of common content".

It can therefore be said that science news repertoires such as that analysed in the present study have the clear pædagogical potential to be used in addressing the NOS in physics classes. Hopefully, this is likely to encourage teachers to use the present repertoire directly in their teaching classrooms or to prepare their own with other items from the press that conform to their teaching interests. Although the repertoire was limited to physics, it nevertheless contains a multitude of items that can be used in teaching other sciences, for instance, those that are related to cross-cutting themes of science or climate change, to name just two.

Finally, it should be stressed that reflexive analysis on NOS using science news items in the press ought to be encouraged as a basic resource in science teacher education programs (both initial and ongoing). Apart from everything noted above, a further reason is that one of the objectives of the competence-forming based framework of education today is that of acquiring the capacity for life-long learning. And for much of the public, both those still in the education system and those who have finished their formal education, science news in the daily press is one of their main sources of information on the progress of science and the issues that scientists are currently interested in. It consequently seems logical that as science teachers we should be prepared to teach already in basic science education how to analyse this type of news in the press critically and reflectively. 
